\title{
Recent observations in surface electromyography recording of triceps brachii muscle in patients and athletes
}

\author{
Md. Asraf Ali*, Kenneth Sundaraj, R. Badlishah Ahmad, Nizam Uddin Ahamed and Md. Anamul Islam \\ AI-Rehab Research Group, Universiti Malaysia Perlis (UniMAP), Kampus Pauh Putra, Perlis, Malaysia
}

\begin{abstract}
.
OBJECTIVE: To observe and analyse the literature on the use of surface electromyography electrodes, including the shape, size, and metal composition of the electrodes used, the interelectrode distance, and the anatomical locations on the muscle at which the electrodes are placed, for the observation of the triceps brachii muscle activity in patients and athletes.

METHODS: We searched the ScienceDirect and SpringerLink online databases for articles published in the English language during the last six years (between January 2008 and December 2013). We specifically searched for the keywords "EMG" and "triceps brachii" in the full text of each of the articles. The inclusion criteria were articles on the use of surface electromyography electrodes to observe the activity of the triceps brachii muscle in patients and athletes.

RESULTS: In the 23 selected articles, the activities of the triceps brachii muscle in a total of 402 subjects were measured using surface electromyography electrodes: 262 subjects in the studies that focused on the rehabilitation of patients with various disorders, and 140 subjects in the studies that focused on the sports performance of various athletes. To record the surface electromyography activity of the triceps brachii muscle, the electrodes were placed over the muscle belly or the three heads (lateral, long, and medial) of the triceps brachii muscle with diverse interelectrode distances. Seventeen studies used bipolar or triode silver/silver chloride electrodes, one study utilised bipolar gold electrodes, one study applied bipolar polycarbonate electrodes, one study used a linear array of four silver bar electrodes, one study utilised DELSYS parallel bar nickel silver electrodes, and two studies did not clearly mention the composition of the electrodes used.

CONCLUSIONS: Bipolar silver/silver chloride circular-shaped electrodes are utilised more frequently than electrodes with a different metal composition and shape. The anatomical locations of the triceps brachii muscle that mainly considered for electrode placement are the lateral, long, and medial heads. A 10-mm electrode size is commonly used to measure the sEMG activity more efficiently. However, we found that an electrode size of up to $40 \mathrm{~mm}$ may be used to reliably measure the sEMG activity on the triceps brachii muscle. A 20-mm interelectrode distance is commonly used to measure the sEMG activity using the above mentioned muscle locations and silver/silver chloride electrodes. We also identified others factors that should be taken into account for the use of the sEMG recording technique on the triceps brachii under real-time conditions.
\end{abstract}

Keywords: Surface electromyography electrodes, triceps brachii muscle activity, rehabilitation; sports

\section{Introduction}

Electromyography (EMG) is a technique that is commonly used to examine the activity of human

\footnotetext{
*Corresponding author: Md. Asraf Ali, AI-Rehab Research Group, Universiti Malaysia Perlis (UniMAP), Kampus Pauh Putra, 02600 Arau, Perlis, Malaysia. Tel.: +60 102549730; Fax: +60 49851695; E-mail: asrafbabu@hotmail.com.
}

muscles during different motor tasks [1]. This technique involves the recording of the electrical activity produced in the muscle and is a useful tool to obtain information on the intensity and time structure of the neuromuscular impulses received by the muscle from the central nervous system [2]. In the EMG technique, the electrical signals of the muscles are measured by the biopotential electrode. Although there 
are several types of biopotential electrodes, electrodes can be divided into two general categories depending on the approaches used to measure the muscle activity: surface electromyography (sEMG) electrodes and intramuscular (iEMG) electrodes [3]. The iEMG electrodes are inserted into muscles to allow the detection of electric potentials close to the muscle fibres, which limits the effect of the volume conductor. As a result, the disadvantage of iEMG electrodes is that these are able to sample only a small number of motor units and consequently cannot be used to obtain data that represent the whole muscle $[4,5]$. In contrast, sEMG electrodes are currently most commonly used to measure muscle activity because these non-invasive electrodes can be placed over the skin, which results in the detection of a general motor unit action potential or a spatial characterisation of the electric potential distribution [6].

Based on the available evidence on the use of sEMG electrodes to measure the activity of upper limb muscles, we found that the activation of the upper limb muscles can also increase the muscle activity during a rhythmic motor task [7]. The upper limb motions play a functional role in balance control with elbow movements [8]. The elbow extension work performed by the triceps brachii muscle (TB), which serves as a powerful extender of the forearm [9-12], has been known for more than a century [13]. However, Salmons [9] demonstrated that the TB is the long muscle on the posterior humerus consisting of a three-headed and fusiform arrangement and operates as a third-class lever because the force is applied between the joint axis and the load [9]. In addition, the three heads of the TB do not necessarily work as a single unit throughout the extension movement [14]. Moreover, Moore and Dalley [15] noted that the TB also plays a role in stabilising the abducted glenohumeral joint by resisting the inferior displacement of the humeral head due to its bi-articular nature. The bi-articular structure of the TB indicates that its length must also be influenced by changes of torque direction [16], and its contribution to elbow joint stability may reduce the injury risk caused by sudden elbow loading [17]. Hollinshead [18] reported the anatomical locations of TB: the long head originates from the infraglenoid tubercle, the lateral head originates from the humerus superior to the radial groove and the lateral intermuscular septum, and the medial head extends from the humerus inferior to the radial groove and the medial intermuscular septum. However, the medial head is mostly covered by

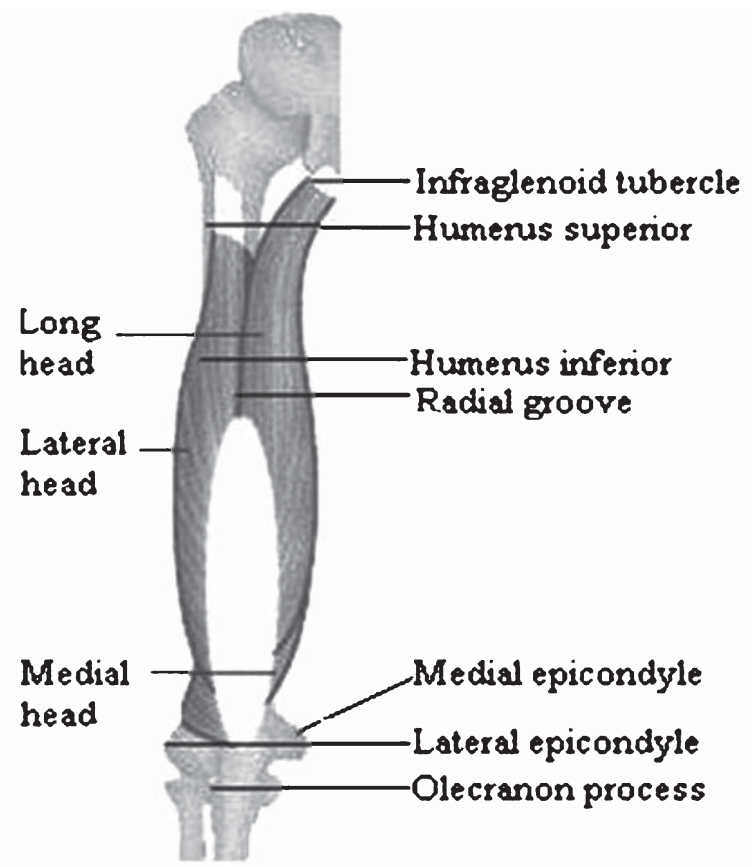

Fig. 1. Anatomical locations of the triceps brachii muscle (left side: mentioning the muscle locations, right side: mentioning the bone locations).

the lateral and long heads and is only visible closer to the elbow joint. Figure 1 shows the anatomical locations on the TB heads that can be used for sEMG recordings.

In addition, shape, size, and metal composition of the electrodes, the interelectrode distance, and anatomical locations of TB are important factors that need to be taken into account during the placement of surface electrodes on the muscles to record EMG signals. Surface Electromyography for the Non-Invasive Assessment of Muscles (SENIAM) [19] recommended that the interelectrode distance between bipolar circular shape surface electrodes of $\mathrm{Ag} / \mathrm{AgCl}$ should be $20 \mathrm{~mm}$ for the placement of electrodes on the long and lateral heads of the TB. But, Emery and Cote [20] recommend an anatomical location $20 \mathrm{~mm}$ medial to the vertical midline of the posterior arm and midway between the acromion and the olecranon process and that an interelectrode distance of $30 \mathrm{~mm}$ should be used for the placement of bipolar surface electrodes on the TB. On the other hand, The DELSYS recommended the parallel bar shape surface electrode of nickel silver to measure the muscles activity. 
Hence, the shape, size, and metallic component of sEMG electrodes, the electrode placement, and the interelectrode distance are factors that are vary between different studies that have recorded the EMG signal on the TB. Therefore, the aim of the present study was to observe the literature regarding the use of sEMG to examine of the condition of the TB during dynamic contraction for the rehabilitation of patients and as the analysis of the sports performance of athletes. The most suitable electrodes types and their structure, the proper interelectrode distances, and the recommended anatomical locations of the TB for the placement of electrodes will be determined in this study. The possibilities for future work on the measurement of the TB activity using sEMG electrodes will also be recommended in the present study.

\section{Methods}

\subsection{Article searching procedure}

We used a systematic searching procedure to identify all of the available articles that discuss the measurement of the TB activity using sEMG electrodes from the ScienceDirect and SpringerLink online digital databases. In our systematic searching procedure, we searched two keywords to search the full text of the article. First, we used the keyword "EMG" to find journal articles published in the English language between the years 2008 and 2013. We then used the keyword "triceps brachii" within the obtained set of results to further narrow the set of analysed publications.

\subsection{Article inclusion and exclusion criteria}

For the final selection of articles that discussed the measurement of the sEMG activity on the TB, we used some criteria to include and exclude articles from the set of articles that were selected through the search of the ScienceDirect and SpringerLink online databases. To include and exclude articles from the set of articles found through our searching procedure, we read the title, abstract, methodology, and results of each article. To determine which articles to include in this study, we considered only those articles that were written in English and that used patient or athlete subjects for the recording of the sEMG signal on the TB. The exclusion criteria were the following: (1) articles that used intramuscular or needle electrode EMG recordings, (2) articles that measured the sEMG of muscles other than TB, (3) articles that addressed the recording of the sEMG signal on the TB of human subjects that were not athletes or patients, and (4) articles that measured the sEMG of non-human subjects.

\subsection{Data extraction}

We carefully read and examined all of the included articles to record the key information. We designed a standard data extraction form for the individual analysis of each article. Two of the authors of the present study (MAA and NUA) used our designed standard data extraction form to record the key information from each of the included articles. The key information that was extracted by both of the authors was compared and evaluated to confirm the accuracy of the extracted records. Each article was evaluated for the following key information: (1) sEMG recording technique, including the electrode type (metal, size, and shape), the interelectrode distance, the sampling and frequency range, and the anatomical locations on the muscle used for the electrode placement, (2) anthropometric variables of the subject, including the subject type, the number of subjects, and the age, gender, height, and weight of the subjects, and (3) the arm selection, the muscle contraction protocol, and the outcomes of the article.

\subsection{Research questions}

The final set of articles was used to answer the following questions: (1) Which types of sEMG electrodes have been utilised to measure the TB activity? (2) Which anatomical locations on the TB have been measured using sEMG electrodes? (3) What are the anatomical locations on the TB that have been used in the literature for the placement of sEMG electrodes?

\section{Results}

\subsection{Article search results}

To collect the relevant articles, we searched for two keywords ("EMG" and "triceps brachii") in the full text of each of the articles. The search of the ScienceDirect and SpringerLink electronic databases using the keyword "EMG" retrieved 18,538 and 6,059 


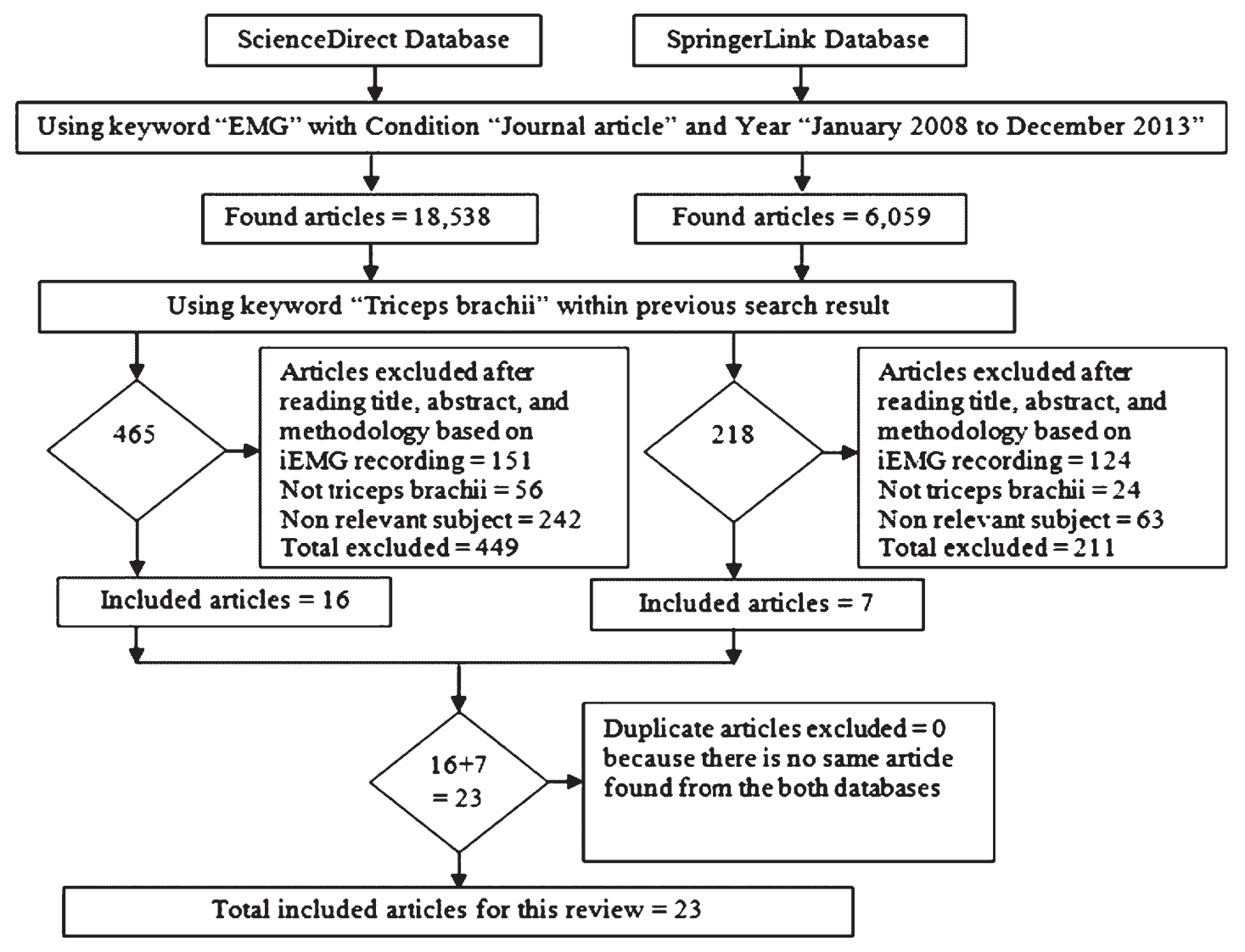

Fig. 2. Articale search results.

articles, respectively. A refined search was then run using the keyword "triceps brachii", and this search resulted in the retrieval of 465 and 218 articles from the ScienceDirect and SpringerLink databases, respectively. We then read the title, abstract, methodology, and results of each article and, based on the inclusion and exclusion criteria, selected 16 articles from the ScienceDirect database and 7 articles from the SpringerLink database. The article search results are summarised in Fig. 2. Thus, as a result of this searching procedure, a total of 23 articles that discussed the measurement of the sEMG activities of the TB were selected for further analysis.

\subsection{Descriptive analysis}

The sEMG recording techniques and contraction protocols used to measure the EMG signal on the TB are presented in Table 1 . The 23 studies that were included measured the sEMG activity on the TB of patients or athletes during the contraction (dynamic and static) of the muscle. The sEMG activity was measured on the TB of a total of 402 subjects, and 262 and 140 subjects were used for the purpose of rehabilitation and sports performance, respectively. The studies on rehabilitation utilised sEMG electrodes to measure the TB activity of patients of stroke [21-24], spinal cord injury [25-28], cerebral palsy [29], gait disorder $[8,30]$, fatigue injury $[20,31]$, and joint stability injury [17] during their trials. In contrast, the sports-based studies applied sEMG electrodes to measure the TB activity of athletes during the following sports activities: tennis [32-34], karate [35, 36], pole vaulting [37], swimming [38, 39], and dart throwing [40]. In addition, the sEMG activities of the TB were compared between healthy subjects and patients [21, 22, 24, 28]. Two other studies [32, 36] also compared the sEMG activities of the muscle between amateur and athlete subjects. A summary of the anthropometric variables of the subjects that were used for the rehabilitation-based and sports-based studies are presented in Tables 2 and 3, respectively. 
Table 1

Techniques and contraction protocols used for the sEMG recording of the TB activity

\begin{tabular}{|c|c|c|c|c|c|}
\hline Reference & Electrode type (diameter) & Frequency $(\mathrm{Hz})$ & Sampling (Hz) & $\begin{array}{l}\text { Electrode } \\
\text { placement }\end{array}$ & Contraction protocol \\
\hline Barker et al. [23] & Bipolar Ag/AgCl $(1 \mathrm{~cm})$ & $30-1,000$ & 2,000 & Lateral head & $\begin{array}{l}\text { During dynamic reaching task (reach } \\
\text { as far as can) and isometric reaching } \\
\text { task (push as hard and as fast as can) }\end{array}$ \\
\hline $\begin{array}{l}\text { Kuhtz-Buschbeck } \\
\text { et al. [30] }\end{array}$ & $\begin{array}{l}\text { Self adhering } \mathrm{Ag} / \mathrm{AgCl} \\
(24 \mathrm{~mm})\end{array}$ & $10-500$ & 1,000 & Long head & $\begin{array}{l}\text { During arm swing while walking on a } \\
\text { treadmill under four conditions: 1) } \\
\text { normal, 2) held, 3) bound, and 4) } \\
\text { anti-normal }\end{array}$ \\
\hline Neto et al. [35] & $\begin{array}{l}\text { Parallel bar bipolar } \\
\mathrm{Ag} / \mathrm{AgCl}(\text { length }=1 \mathrm{~cm}, \\
\text { width }=0.2 \mathrm{~cm}, \\
\text { interelectrode } \\
\text { distance }=1 \mathrm{~cm})\end{array}$ & $50-500$ & 3,500 & $\begin{array}{l}\text { Lateral or long } \\
\text { head }\end{array}$ & $\begin{array}{l}\text { During the performance of Kung Fu } \\
\text { Yau-Man palm strike without impact }\end{array}$ \\
\hline $\begin{array}{l}\text { Bazzucchi } \\
\text { et al. [32] }\end{array}$ & $\begin{array}{l}\text { Linear array of four silver } \\
\text { bar (length }=5 \mathrm{~mm} \text {, } \\
\text { thickness }=1 \mathrm{~mm} \text {, } \\
\text { interelectrode } \\
\text { distance }=10 \mathrm{~mm})\end{array}$ & $10-450$ & 2,048 & Muscle belly & $\begin{array}{l}\text { During 1) maximal voluntary isometric } \\
\text { contraction; 2) maximal flexion and } \\
\text { extension isokinetic concentric } \\
\text { contractions at } 15^{\circ}, 30^{\circ}, 60^{\circ}, 120^{\circ} \text {, } \\
180^{\circ} \text {, and } 240^{\circ} / \mathrm{s} ; 3 \text { ) maximal flexion } \\
\text { and extension isokinetic eccentric } \\
\text { contractions at } 15^{\circ} / \mathrm{s}\end{array}$ \\
\hline Ikuta et al. [39] & Bipolar gold (5 mm) & $20-200$ & 500 & Muscle belly & $\begin{array}{l}\text { During a } 200-\mathrm{m} \text { front crawl swimming } \\
\text { without any turns }\end{array}$ \\
\hline Rota et al. [33] & Triode $\mathrm{Ag} / \mathrm{AgCl}(2 \mathrm{~cm})$ & $10-500$ & 2,048 & $\begin{array}{l}\text { Lateral or long } \\
\text { head }\end{array}$ & $\begin{array}{l}\text { During the performance of five series } \\
\text { of ten crosscourt forehand drives }\end{array}$ \\
\hline $\begin{array}{l}\text { Rogowski } \\
\text { et al. [34] }\end{array}$ & Triode $\mathrm{Ag} / \mathrm{AgCl}(2 \mathrm{~cm})$ & $10-500$ & 2,048 & $\begin{array}{l}\text { Lateral or long } \\
\text { head }\end{array}$ & $\begin{array}{l}\text { During the performance of seven series } \\
\text { of ten crosscourt forehand drives }\end{array}$ \\
\hline $\begin{array}{l}\text { Pijnappels } \\
\text { et al. [8] }\end{array}$ & Bipolar $\mathrm{Ag} / \mathrm{AgCl}$ & & 1,000 & Muscle belly & $\begin{array}{l}\text { During arm swing while walking under } \\
\text { normal and obstacle-filled conditions }\end{array}$ \\
\hline Emery et al. [20] & Bipolar Ag/AgCl $(1 \mathrm{~cm})$ & $20-500$ & 1,080 & Medial head & $\begin{array}{l}\text { During a shoulder angular position } \\
\text { sense task and an upper limb } \\
\text { endpoint position sense task }\end{array}$ \\
\hline $\begin{array}{l}\text { Brændvik } \\
\text { et al. [29] }\end{array}$ & Polycarbonate $\left(10 \mathrm{~mm}^{2}\right)$ & $20-450$ & 1,000 & Lateral head & $\begin{array}{l}\text { During elbow flexion-extension with } \\
\text { maximal voluntary isokinetic } \\
\text { concentric contraction, passive } \\
\text { isokinetic movement, and } \\
\text { sub-maximal isometric force tracing }\end{array}$ \\
\hline $\begin{array}{l}\text { VencesBrito et al. } \\
\text { [36] }\end{array}$ & Bipolar $\mathrm{Ag} / \mathrm{AgCl}$ & $10-400$ & 1,600 & Lateral head & $\begin{array}{l}\text { During a karate punching movement } \\
\text { (choku-zuki) on a fixed target } \\
\text { (makiwara) }\end{array}$ \\
\hline Frère et al. [37] & Bipolar Ag/AgCl (10 mm) & $10-700$ & 2,500 & Lateral head & $\begin{array}{l}\text { During the performance of between } 5 \\
\text { and } 10 \text { vaults at } 90 \% \text { of the athlete's } \\
\text { best performance }\end{array}$ \\
\hline Lohse et al. [40] & Bipolar Ag/AgCl $(1 \mathrm{~cm})$ & $20-500$ & 1,000 & Long head & During dart throwing \\
\hline $\begin{array}{l}\text { Janssen-Potten } \\
\text { et al. [25] }\end{array}$ & Bipolar $\mathrm{Ag} / \mathrm{AgCl}\left(1 \mathrm{~cm}^{2}\right)$ & & & $\begin{array}{l}\text { Lateral and long } \\
\text { head }\end{array}$ & $\begin{array}{l}\text { During a standardised arm and hand } \\
\text { function task with a wheelchair }\end{array}$ \\
\hline Stirn et al. [38] & Bipolar Ag/AgCl (9 mm) & $16-500$ & 2,000 & Long head & $\begin{array}{l}\text { During a } 100-\mathrm{m} \text { front crawl swim at a } \\
\text { maximum perceived effort level }\end{array}$ \\
\hline Holmes et al. [17] & Bipolar $\mathrm{Ag} / \mathrm{AgCl}$ & $10-1,000$ & 2,048 & Muscle belly & $\begin{array}{l}\text { During elbow extension using a } \\
\text { combination of three body postures } \\
\text { (standing, supine, and sitting) and } \\
\text { three hand load conditions (none, } \\
\text { solid, and fluid) }\end{array}$ \\
\hline Serrao et al. [21] & Bipolar Ag/AgCl (1 cm) & $10-400$ & 1,000 & $\begin{array}{l}\text { Lateral or long } \\
\text { head }\end{array}$ & $\begin{array}{l}\text { During the following movement: } \\
\text { reaching out from a starting position, } \\
\text { picking up a cylinder, and returning } \\
\text { it to the starting position }\end{array}$ \\
\hline
\end{tabular}


Table 1

(Continued)

\begin{tabular}{|c|c|c|c|c|c|}
\hline Reference & Electrode type (diameter) & Frequency $(\mathrm{Hz})$ & Sampling $(\mathrm{Hz})$ & $\begin{array}{l}\text { Electrode } \\
\text { placement }\end{array}$ & Contraction protocol \\
\hline Fang et al. [22] & & $10-500$ & 1,000 & & $\begin{array}{l}\text { During elbow extension in a reaching } \\
\text { task }\end{array}$ \\
\hline Li et al. [24] & Bipolar Ag/AgCl & $10-500$ & 2,000 & $\begin{array}{l}\text { Lateral, long, and } \\
\text { medial head }\end{array}$ & $\begin{array}{l}\text { During voluntary elbow flexion in a } \\
\text { vertical plane }\end{array}$ \\
\hline Rankin et al. [26] & & & & Long head & $\begin{array}{l}\text { During four conditions of wheelchair } \\
\text { propulsion: 1) self-selected } \\
\text { propulsion, 2) minimising cadence, } \\
\text { 3) maximal contact angle, and 4) } \\
\text { minimum peak force in biofeedback }\end{array}$ \\
\hline Huang et al. [27] & $\begin{array}{l}\text { Parallel bar nickel silver } \\
\text { (length }=1 \mathrm{~cm} \\
\text { width }=1 \mathrm{~cm} \text {, } \\
\text { interelectrode distance }=1 \text { ) }\end{array}$ & & & Lateral head & $\begin{array}{l}\text { During recumbent stepping using } \\
\text { active, passive, and resting arm } \\
\text { efforts }\end{array}$ \\
\hline Louis et al. [28] & Bipolar Ag/AgCl $(4 \mathrm{~cm})$ & $6-1,600$ & & Long head & $\begin{array}{l}\text { During wheelchair propulsion using } \\
\text { self-selected speed in twelve } \\
\text { wheelchair configurations }\end{array}$ \\
\hline Yung et al. [31] & Bipolar Ag/AgCl $(1 \mathrm{~cm})$ & $10-1,000$ & 2,048 & $\begin{array}{l}\text { Lateral, medial } \\
\text { head }\end{array}$ & $\begin{array}{l}\text { During the following: } 1 \text { ) isometric } \\
\text { elbow extension at } 15 \% \text { maximum } \\
\text { voluntary contraction (MVC), } 2 \text { ) } \\
\text { intermittent elbow extension } \\
\text { alternating between } 0 \text { and } 30 \% \\
\text { MVC, } 3 \text { ) elbow extension alternating } \\
\text { between } 7.5 \text { and } 22.5 \%, 4 \text { ) elbow } \\
\text { extension alternating between } 1 \text { and } \\
29 \% \text { MVC, and 5) intermittent } \\
\text { sinusoidal wave pattern with peaks at } \\
0 \text { and } 30 \% \text { MVC }\end{array}$ \\
\hline
\end{tabular}

\subsection{Research question 1: Which types of sEMG electrodes have been utilised to measure the $T B$ activity?}

Seventeen of the studies used bipolar or triode silver/silver chloride $(\mathrm{Ag} / \mathrm{AgCl})$ sEMG electrodes, one study [39] utilised bipolar gold sEMG electrodes, and one study [29] applied polycarbonate sEMG electrodes for the recording of the EMG activity on the TB. In addition, one study used a linear array of four silver bar sEMG electrodes [32]. Moreover, one study [27] referred to "DELSYS", which are parallel bar nickel silver sEMG electrodes, and two studies $[22,26]$ did not clearly mention the composition of the sEMG electrodes used.

\subsection{Research question 2: Which anatomical locations on the TB have been measured with sEMG electrodes?}

Of the 23 selected studies, eighteen recorded the heads of the TB, whereas the four studies $[8,17,32,39]$ focused the muscle belly of TB, and one study [22] did not mention the specific anatomical location on the TB used for the sEMG recordings. Of the 18 studies that analysed the TB heads, 12 studies [21, 23-25, 27, 29, $31,33-37$ focused on the lateral head, 11 studies [21, 24-26, 28, 30, 33-35, 38, 40] analysed the long head, and three studies $[20,24,31]$ focused on the medial head. Moreover, some of the studies simultaneously measured the sEMG electrodes activity from the three heads (lateral, long, and medial) [24], from the lateral and medial heads [31], and from the lateral and long heads $[21,25,33-35]$ of the TB.

\subsection{Research question 3: What are the anatomical locations on the TB that have been used in the literature for the placement of sEMG electrodes?}

There was no stability regarding the anatomical location on the TB used for the placement of the sEMG electrodes. Only two studies $[22,26]$ did not present the anatomical locations on the muscle used for the electrode placement, and one study [8] only referred to another study regarding the anatomical location on 
Table 2

Summary of the anthropometric variables of the participants used in the rehabilitation-based studies

\begin{tabular}{|c|c|c|c|c|c|c|c|c|c|c|c|c|}
\hline \multirow[t]{2}{*}{ Rehabilitation } & \multirow[t]{2}{*}{ Reference } & \multirow[t]{2}{*}{$n$} & \multicolumn{5}{|c|}{ Patient } & \multicolumn{5}{|c|}{ Healthy } \\
\hline & & & $\mathrm{M}(n)$ & $\mathrm{F}(n)$ & $\mathrm{H}(\mathrm{cm})$ & $\mathrm{W}(\mathrm{kg})$ & AA (year) & $\mathrm{M}(n)$ & $\mathrm{F}(n)$ & $\mathrm{H}(\mathrm{cm})$ & W (kg) & AA (year) \\
\hline \multirow[t]{4}{*}{ Stroke } & Serrao et al. [21] & 16 & 8 & & & & 60 & 8 & & & & 53 \\
\hline & Fang et al. [22] & 29 & 21 & & & & 59 & 5 & 3 & & & 60 \\
\hline & Barker et al. [23] & 42 & 42 & & & & 26 & & & & & \\
\hline & Li et al. [24] & 8 & 3 & 1 & & & 51 & 1 & 3 & & & 28 \\
\hline \multirow[t]{4}{*}{ Spinal cord injury } & Rankin et al. [26] & 13 & 11 & 2 & 171 & 69 & 33 & & & & & \\
\hline & Huang et al. [27] & 15 & 9 & 6 & & & 50 & & & & & \\
\hline & Louis et al. [28] & 20 & 10 & & 169 & 70 & 29 & 10 & & 175 & 71 & 23 \\
\hline & Janssen-Potten et al. [25] & 20 & & & & & & & & & & \\
\hline Cerebral palsy & Brændvik et al. [29] & 21 & 9 & 12 & & & 13 & & & & & \\
\hline \multirow[t]{2}{*}{ Gait disorder } & Kuhtz-Buschbeck et al. [30] & 20 & & & & & & 20 & & 185 & 80 & 29 \\
\hline & Pijnappels et al. [8] & 10 & & & & & & 6 & 4 & 179 & 73 & 25 \\
\hline \multirow[t]{2}{*}{ Fatigue } & Emery et al. [20] & 18 & & & & & & 9 & 9 & & & 23 \\
\hline & Yung et al. [31] & 15 & & & & & & 15 & & 178 & 76 & 24 \\
\hline Joint stability & Holmes et al. [17] & 15 & & & & & & 15 & & 179 & 81 & 26 \\
\hline
\end{tabular}

$n=$ number of subject, $\mathrm{M}=$ male, $\mathrm{F}=$ female, $\mathrm{H}=$ height, $\mathrm{W}=$ weight, $\mathrm{AA}=$ average age.

Table 3

Summary of the anthropometrics variables of the participants used in the sports-based studies

\begin{tabular}{|c|c|c|c|c|c|c|c|c|c|c|c|c|}
\hline \multirow[t]{2}{*}{ Sport } & \multirow[t]{2}{*}{ Reference } & \multirow[t]{2}{*}{$n$} & \multicolumn{5}{|c|}{ Professional } & \multicolumn{5}{|c|}{ Amateur } \\
\hline & & & $\mathbf{M}(n)$ & $\mathrm{F}(n)$ & $\mathrm{H}(\mathrm{cm})$ & W (kg) & AA (year) & $\mathrm{M}(n)$ & $\mathrm{F}(n)$ & $\mathrm{H}(\mathrm{cm})$ & W (kg) & AA (year) \\
\hline \multirow[t]{3}{*}{ Tennis } & Bazzucchi et al. [32] & 18 & 8 & & 180 & 78 & 22 & 10 & & 178 & 73 & 25 \\
\hline & Rota et al. [33] & 21 & 21 & & 178 & 71 & 23 & & & & & \\
\hline & Rogowski et al. [34] & 15 & 15 & & 177 & 70 & 23 & & & & & \\
\hline \multirow[t]{2}{*}{ Karate } & Neto et al. [35] & 8 & & & 176 & 75 & $<18$ & & & & & \\
\hline & $\begin{array}{l}\text { VencesBrito } \\
\text { et al. [36] }\end{array}$ & 28 & 10 & 8 & & & 15 & 9 & 1 & & & \\
\hline Pole vaulting & Frère et al. [37] & 7 & & & 180 & 74 & & & & & & \\
\hline \multirow[t]{2}{*}{ Swimming } & Stirn et al. [38] & 11 & 11 & & 185 & 77 & 22 & & & & & \\
\hline & Ikuta et al. [39] & 20 & 20 & & 175 & 68 & 20 & & & & & \\
\hline Dart throwing & Lohse et al. [40] & 12 & & & & & & & & & & \\
\hline
\end{tabular}

$n=$ number of subject, $\mathrm{M}=$ male, $\mathrm{F}=$ female, $\mathrm{H}=$ height, $\mathrm{W}=$ weight, $\mathrm{AA}=$ average age.

the TB used for the electrode placement protocol. In contrast, other studies reported the anatomical locations of the TB used for electrode placement and the specific interelectrode distance. The different electrode placements on the anatomical locations on the TB used for sEMG recording are the following:

\subsubsection{Lateral head of the TB}

The bipolar $\mathrm{Ag} / \mathrm{AgCl}$ sEMG electrodes have been placed on the level of the lateral head of the TB with interelectrode distances of $10 \mathrm{~mm}$ [35], $20 \mathrm{~mm}$ [23, 24, 31, 33, 34, 36, 37], $23 \mathrm{~mm}$ [25]. The other studies placed single differential polycarbonate sEMG electrodes [29] and parallel bar nickel silver sEMG electrodes [27] using an interelectrode distance of $20 \mathrm{~mm}$. With respect to the anatomical location on the lateral head of the TB, nine of the studies $[21,23,25$, 28, 29, 33-35, 37] followed the SENIAM guidelines described by Hermens et al. [41, 48], one of the studies [36] used the instructions provided by Basmajin and De Luca [2], one of the studies [24] applied the recommendations given by Cram et al. (1998) [45], one of the studies [27] specified the muscle belly along the long axis, and one of the studies [31] indicated the muscle belly for the purpose of electrode placements.

\subsubsection{Long head of the TB}

The bipolar or triode $\mathrm{Ag} / \mathrm{AgCl}$ sEMG electrodes have been placed on the level of the long head of the TB with interelectrode distances of $10 \mathrm{~mm}[35$, 40], $20 \mathrm{~mm}$ [24, 33, 34, 38], $23 \mathrm{~mm}$ [25], and $25 \mathrm{~mm}$ [28]. With respect to the anatomical location on the long head of the TB, eight of the studies [21, 25, 28, 30, 33-35, 38] followed the SENIAM guidelines reported by Hermens et al. (1997 and 2000) [41, 48], and one of the studies [24] used the recommendations 
given by Cram et al. (1998) [45] for electrode placements.

\subsubsection{Medial head of the TB}

The bipolar $\mathrm{Ag} / \mathrm{AgCl}$ sEMG electrodes have been placed on the level of the medial head of the TB with interelectrode distances of $30 \mathrm{~mm}$ [20] and $20 \mathrm{~mm}$ [24, 31]. With respect to the anatomical location on the medial head of the TB, one of the studies [20] mentioned a location $20 \mathrm{~mm}$ medial to the vertical midline of the posterior arm and midway between the acromion and the olecranon, one of the studies [24] followed the recommendations given by Cram et al. (1998) [45], and one of the studies [31] noted the muscle belly for the purpose of electrode placement.

\subsubsection{Muscle belly of the TB}

The bipolar $\mathrm{Ag} / \mathrm{AgCl}$ sEMG electrodes have been placed on the muscle belly of the TB with an interelectrode distance of $25 \mathrm{~mm}$ [17], and the study [8] did not provide the interelectrode distance. The other studies placed a linear array of four silver bar sEMG electrodes using an interelectrode distance of $10 \mathrm{~mm}$ [32], and bipolar gold sEMG electrodes with an interelectrode distance of $20 \mathrm{~mm}$ [39] on the muscle belly of the TB. With respect to the anatomical location on the muscle belly of the TB, one of the studies [32] noted that the electrodes were placed along a line connecting the acromion to the olecranon, one of the studies [39] noted the muscle belly recommendation provided by Perotto [44], one of the studies [8] identified a location in line with the muscle fibre, and one of the studies [17] mentioned a location in line with the muscle fibre direction and between the innervations zone and the terminal tendon.

\section{Discussion}

The present study provides a summary of the data found in the literature that address the use of sEMG electrodes for the evaluation of TB activities during dynamic contraction for the rehabilitation of patients and the analysis of the sports performance of athletes. To measure the TB activities with the use of sEMG electrodes, we chose patients for the condition of slow movement and athlete subjects for the condition of fast movement of dynamic contraction. Different topics, such as the anatomical locations on the TB used for the recordings, the application of elec- trodes, including their shapes, sizes, and composition of different metals, the interelectrode distances, and the electrode placements on the muscle, were discussed. All of the included articles mostly evaluated the TB activity through sEMG electrode recordings of the subjects, but there were significant dissimilarities in the protocols used to record these signals. Therefore, it is not possible to compare the results of the included studies due to the variations in the protocols.

\subsection{Materials, shape, and size of surface electromyography electrodes}

A sEMG electrode is a sensor for measuring the electrical activity of a muscle which is a non-invasive tool for the assessment of the neuromuscular system [41]. Presently, sEMG electrodes are more popular for the measurement of EMG activity due to the easiness of their placement on the superficial muscle. sEMG electrodes composed of various metals have been applied to measure the TB activity in several of the studies that are reported in the present study. Based on these studies, we can classify sEMG electrodes based on their composition of various metals: $\mathrm{Ag} / \mathrm{AgCl}[23,30$, 35], gold [39], silver [32], nickel silver [27], and polycarbonate [29]. Most of the studies included in this study applied a bipolar surface electrode of $\mathrm{Ag} / \mathrm{AgCl}$ recording system for the measurement of TB activity because the $\mathrm{Ag} / \mathrm{AgCl}$ metallic electrodes is highly stable and present the lowest noise interface with respect to other metallic electrodes [41]. Usually, the EMG signals are measured from the electrodes detection area on the skin which depends on electrodes shape and its size. In the present study, most of the included literatures applied circular-shaped $\mathrm{Ag} / \mathrm{AgCl}$ electrodes, although a few studies utilised parallel bar-shaped electrodes composed of $\mathrm{Ag} / \mathrm{AgCl}$ [35], silver [32], and nickel silver [27] to measure the EMG activity of the TB. In addition, one study [39] applied circular-shaped electrodes composed of gold, and the other study [29] utilised square-shaped single differential polycarbonate electrodes to measure the EMG activity of the TB. Two other studies $[22,26]$ did not provide details on the metals and the shape of the electrodes used for the EMG measurements on the TB. However, four studies [23, 27, 29, 35] measured the EMG activity of the same anatomical location of the TB (lateral head) utilising different types of electrodes, namely circular-shaped (bipolar $\mathrm{Ag} / \mathrm{AgCl}$, and single differential polycarbonate) and parallel bar-shaped (bipolar 
$\mathrm{Ag} / \mathrm{AgCl}$, and nickel silver) electrodes. The authors of the study [35] utilized the bipolar superficial electrodes consisting of two rectangular parallel bars of $\mathrm{Ag} / \mathrm{AgCl}(1 \mathrm{~cm}$ in length, $0.2 \mathrm{~cm}$ in width, and separated by $1 \mathrm{~cm}$ ) and coupled to a rectangular acrylic resin capsule 2.2 in length, $1.9 \mathrm{~cm}$ in width and $0.6 \mathrm{~cm}$ height with an internal amplifier (with gain of 20) in order to reduce the effects of electromagnetic interference and other noise. On the other hand, the authors of the study [29] applied bipolar superficial electrodes consisting of circular shaped of single differential polycarbonate $\left(1 \mathrm{~cm}^{2}\right.$ area, and $1 \mathrm{~cm}$ spacing) to record the EMG signals of TB. We cannot compare the effect on the EMG signal recording between circular and parallel bar shaped electrodes because of the different electrode metal applied for recording. With shape of the electrodes, SENIAM has not given any proper guidelines or instructions to record the EMG activity on the TB. But, the sEMG recording signals could be varied depends on the electrode shape during dynamic contractions. Because the shape of the electrode locates the surface area of the muscle fibre to detect the motor unit action potentials which is the major factor to produce the sEMG signals. These signals are not free of noise and crosstalk from the adjacent muscles. Thus, the shape of the electrode in sEMG recording technique during dynamic contraction needs for further research to reduce crosstalk from adjacent muscles and skin movement relative to the underlying motor units.

With the respect of electrodes size, different studies applied different sizes of circular-shaped (diameters ranging from $5 \mathrm{~mm}$ to $40 \mathrm{~mm}$ ) surface electrodes composed of $\mathrm{Ag} / \mathrm{AgCl}$ or other metals (as defined above) for the measurement of the EMG activity on the superficial layer of the TB. The electrodes size has been reached in the literature $[8,17,22,26,36]$ without any conclusion. However, fourteen of the studies $(60.87 \%)$ utilised an electrode size of up to $10 \mathrm{~mm}$, and four of the studies $(17.39 \%)$ [28, 30, 33, 34] applied electrodes greater than $10 \mathrm{~mm}$ in size to measure the EMG activity of the TB. For example, Dimitrova et al. [42] utilised square-shaped electrode plates of different sizes $(1 \times 1 \mathrm{~mm}, 2 \times 2 \mathrm{~mm}, 4 \times 4 \mathrm{~mm}, 8 \times 8 \mathrm{~mm}$, $10 \times 10 \mathrm{~mm}$, and $20 \times 20 \mathrm{~mm}$ ) and concluded that a greater electrode size measured less motor unit potentials due to the spatial filtering of the electrode surface. Furthermore, SENIAM recommends that the electrodes size for the direction of the muscle fibres should not exceed $10 \mathrm{~mm}$ because an increase in the size in the direction of the muscle fibres can exert an integrative effect on the surface EMG signal and decrease the highfrequency content [41]. Although, the reliable sEMG activity for the TB can be obtained with an electrode size up to $40 \mathrm{~mm}[28,30,33,34]$, but smaller-sized electrodes might be better to measure the EMG activity on the TB because a smaller electrode will results in a greater real-time EMG activity due to fast motion of the muscle, even under slow motion conditions.

\subsection{Anatomical location and interelectrode distance for the placement of surface electromyography electrodes}

In this present study, a large number of different anatomical locations and interelectrode distances were found for electrode placement on the TB. With respect to the anatomical locations, it was not possible to determine the electrode placement in a few of the studies $[8,26]$ due to the insufficient information provided. For example, Pijnappels et al. [8] noted the location "over the muscle belly in line with the muscle fibres" but did not specify the lateral distance to the acromion and the olecranon process, and Rankin et al. (2009) [26] reported the location as "long head". Holmes and Keir [17] used another anatomical location for the electrode placement: the muscle belly in line with muscle fibre direction and between the innervations zone and the terminal tendon. However, Sommerich et al. [43] reported two approaches for the location of the surface electrodes: a muscle-specific site and a location-specific site. A study [27] placed the electrodes "over the muscle belly along the long axis", which is an example of a muscle-specific site. Emery and Cote [20] placed the electrodes at a position " $20 \mathrm{~mm}$ medial to the vertical midline of the posterior arm and midway between the acromion and the olecranon process", which is an example of a locationspecific site. Most of the electrode placement reports in the analysed studies were location-specific or a combination of both approaches. For example, the studies $[21,23,25,28-30,33-35,37,38]$ determined the electrodes placement on the TB according to the SENIAM guidelines: (1) with respect to the long head, the electrodes need to be placed two-finger-widths medial to the line that is halfway between the posterior cristae of the acromion and the olecranon, and, (2) with respect to the lateral head, the electrodes need to be placed twofinger-widths lateral to the line that is halfway between the posterior cristae of the acromion and the olecranon [19]. Ikuta et al. [39] and Li et al. [24] placed the 
electrodes on the TB based on the recommendations provided by Perotto [44] and Cram et al. [45], respectively. Perotto [44] and Cram et al. [45] recommended that the electrodes should be placed at the midpoint of the contracted muscle belly of the lateral, long, and medial heads of the TB. Thus, we concluded that the medial head may be another anatomical location for the placement of electrodes to measure the EMG activity of the TB that is not recommended by SENIAM. Furthermore, Sommerich et al. [43] suggested that location-specific sites could be suitable when focusing on the levels of activity in a muscle group, but crosstalk from an adjacent muscle is more likely in this type of site than in a muscle-specific location.

With respect to the interelectrode distance for the placement of sEMG electrodes on the TB, we were unable to conclude the interelectrode distance used by the studies $[22,26]$ for the placement of sEMG electrodes because of insufficient information provided. In the present study, we found that TB activities were measured using interelectrode distances that ranged from 10 to $30 \mathrm{~mm}$ in the literatures, and most of the studies $(73.91 \%)$ followed the SENIAM guidelines for electrode placement. SENIAM defined the interelectrode distance as the centre-to-centre distance between the conductive areas of two bipolar electrodes and recommended an interelectrode distance of $20 \mathrm{~mm}$. However, the TB has been measured by placing electrodes as follows: with an interelectrode distance of less than $20 \mathrm{~mm}[27,29,35]$ and greater than of $20 \mathrm{~mm}$ [25] on the lateral head, with an interelectrode distance of less than $20 \mathrm{~mm} \mathrm{[35]} \mathrm{and} \mathrm{greater} \mathrm{than} 20 \mathrm{~mm} \mathrm{[25,}$ 28] on the long head, with an interelectrode distance greater than $20 \mathrm{~mm} \mathrm{[20]} \mathrm{on} \mathrm{the} \mathrm{medial} \mathrm{head,} \mathrm{and} \mathrm{with}$ an interelectrode distance of less than $20 \mathrm{~mm} \mathrm{[32]} \mathrm{and}$ greater than $20 \mathrm{~mm}$ [17] on the muscle belly in line with the muscle fibre direction and between the innervations zone and the terminal tendon. Mesin et al. [46] noted that the EMG amplitude is relatively narrow, particularly if the interelectrode distance is comparable to the fibre semi-length and if the electrodes are placed 25 to $40 \mathrm{~mm}$ away from the innervations zone, and concluded that the interelectrode distance must be small with respect to the distance between the innervations zone and the tendon. Kamen and Gabriel [47] distinguished a markedly lower EMG amplitude with an interelectrode distance of less than $5 \mathrm{~mm}$. Blok and Stegeman (1997) [48] recommended the use of an interelectrode distance of up to $20 \mathrm{~mm}$ to measure the maximal EMG signal amplitude. Hence, the interelectrode distance could be considered between 5 to $20 \mathrm{~mm}$ for the placement of electrodes to measure the maximal EMG amplitude.

\subsection{Sampling rate and frequency range for the use of surface electromyography}

The sampling rate and frequency range is another challenge to ensure avoiding the loss of information from the sEMG signal on the TB. Merletti et al. [49] reported that the entire sEMG frequency bandwidth should be in the range of 10 to $500 \mathrm{~Hz}$ for the measurement of the electrode-skin impedance, and sampling frequencies commonly used 1024 or $2048 \mathrm{~Hz}$ for sEMG recording system. However, to avoid loss of information from the sEMG signals, the sampling rate should be at least double the highest frequency range of the signals [50]. In this present study, we did not find any literature that applied less sampling rate than double the highest frequency range (Table 1), and although most of the studied recorded sEMG signals from the TB at sampling rate of less than $2048 \mathrm{~Hz}$ (Table 1), the sEMG signals on the TB might be recorded at a sampling rate well above this range during dynamic contraction [35]. In contrast, the sEMG bandwidth might be considered for the measurement of the TB activity at a frequency range well above the range of the highest frequency bandwidth [17, 23, 28, 31, 37] during dynamic contraction. However, the interpretation of the evaluation of the EMG frequency over time is remained difficult during dynamic contraction [51, 52]. Thus, it could be interesting to define specific recommendations and guidelines for the sampling rate and frequency range of sEMG recording signals that should be optimally used for the real-time measurement of the EMG activity on skeletal muscle due to motion of the muscle during dynamic contractions.

\subsection{Dynamic contraction protocols used for surface electromyography recording on the $T B$}

The recording values of the sEMG on the TB need to be as accurate and reliable as possible if they are to be used for the rehabilitation of patients and the analysis of the sports performance of athletes. In the real-time situation, the recording EMG value varies depending on the contraction of the muscle. To measure the TB activity, the EMG values need to be recorded in isometric and/or dynamic contraction of the TB. Usually, the 
length of the muscle changes during both isometric and dynamic contraction. In these contractions, the muscle length is shortened during concentric contraction and lengthens during eccentric contraction. But, the joint angle does not change during isometric contractions, and does during dynamic contractions. Thus, the sEMG recording techniques on the TB could be varied between isometric and dynamic contractions due to the variation in the muscle structure and its motion during these different contractions. However, SENIAM has recommended the use of sEMG recording methodology, such as the metal composition of the electrode, the electrode size, the anatomical locations used for the placement of the electrodes on the muscle, and the interelectrode distance, for isometric contraction. In this study, more than $65 \%$ of the literatures used the electrode metal and size and the anatomical location for electrode placement recommended by SENIAM to record the EMG values from the TB during dynamic contraction, whereas less than $27 \%$ of the studies [23, 24, 31, 36-38] followed SEMIAN recommendation for the interelectrode distance. Although SENIAM recommended the interelectrode distance for isometric contraction to record the sEMG signals from the various muscle but it might not be the same for dynamic contraction because the interelectrode distance is a fact to obtain a large sEMG signal and consequently noise of the signal. Hence, the interelectrode distance may be a challenge in the sEMG recording techniques due to change the muscle length and motion of the TB during dynamic contraction.

Particularly, at the high force and velocities for dynamic contraction, the frequency component of the signal is very sensitive to the morphological properties of the muscle and the relative relationship of the EMG electrodes to the neuromuscular system [53-55]. In contrast, the EMG values have been shown to vary with the muscle length and thus the joint angle [56] and with the applied torque and muscle length but not with the velocity during dynamic contraction [57]. In addition, muscle movement is another factor that introduces variability into the sEMG recording that complicates the detection and interpretation of the signal, i.e., a shortening of the muscle fibres results in a shift between the muscle fibres and the detection system [58]. Thus, the length, torque, joint angle of elbow and shoulder, level of force and velocity, and motion of the TB are factors that need to be taken into account for the recording of the EMG values of the TB during dynamic contraction.

\subsection{Recommendations for $s E M G$ recording techniques on the TB during dynamic contraction}

On the above mentioned challenges for measuring the sEMG signal during dynamic contractions, following circumstances are essential for recording the reliable and noise free signal from the $\mathrm{TB}$, those are suggested bellow:

(1) Electrode placement site on TB: According to the previous literatures, the lateral, long, and medial heads of TB are the anatomical location for placing electrodes during several dynamic movements. However, researchers yet did identify at which head can generate maximum signal amplitude during continuous contraction activities by the TB (for example, isokinetic exercise during swimming). Moreover, none of the literature mentioned any recommendation to reduce the signal crosstalk between the adjacent muscles while multiple electrodes are placed on TB. Therefore, these essential issues need to be assessing for SEMG signal analysis on patients and the athletes. Because different electrode locations over the same muscle can present signals with significantly different feature.

(2) The metal of the electrode: Most of the researchers applied the metal of $\mathrm{Ag} / \mathrm{AgCl}$ electrodes to record the sEMG activity because it can establish a good relationship between skin and electrode to pass the electric signal more frequently, and also it can reduce the electrical noises compared with other metals electrode (for example, gold, silver, nickel silver, and polycarbonate).

(3) The size of the electrode: A number of TB related articles preferred to use an electrode between the diameters of $1 \mathrm{~mm}$ to $40 \mathrm{~mm}$. Also, a recommendation made by SENIAM regarding this issue as it should be better if the electrode size is not more than $10 \mathrm{~mm}$. It has also proved by the researchers that, smaller electrode can measures more motor unit potentials and able to produce maximum signal value than the larger electrode. However, further EMG oriented assessments are needed during different motion related activities of TB.

(4) Interelectrode distance on TB: in the entire literatures, researchers chosen the interelectrode distances between $10-\mathrm{mm}$ to $30-\mathrm{mm}$. This measurement ration also showed up while some researchers recommended the inter electrode distance should not be less than $5 \mathrm{~mm}$ and greater than $20 \mathrm{~mm}$ to get the maximum signal amplitude. But, no research indicated the exact distance between the electrodes within this 5 to $20 \mathrm{~mm}$ 
space which can generate maximum signal amplitude and also able to reduce the crosstalk between the adjacent muscle. Because the length of the muscle fibre able to shorten and lengthen the TB during its contraction by the flexion and extension phenomenon.

(5) The shape of the electrode: According to the previous literatures, most of the experiments were done with the following electrode shapes: the circular, the squared and the rectangular parallel bar. Although researchers have utilized these shape of electrodes but nobody has defined any recommendation for exact electrode shape. To improve the signal quality and reduce the signal interference during dynamic contractions of TB, the shape of the electrode needs to be assessing for the sEMG recording. Because the electrode shape locates the surface area of the muscle fibre to detect the motor unit action potentials which is the major factor of the electrical signal of a muscle.

\section{Conclusions}

The aim of the present study was to observe the literature regarding the use of sEMG to examine the condition of the TB for the rehabilitation of patients and as the analysis of the sports performance of athletes. For this purpose, the EMG values from the TB need to be recorded during dynamic movement. However, it is a challenging process to examine the TB activity during dynamic movement through sEMG recordings. Based on the articles analysed, our observation reveals the following: (1) for both rehabilitation and sports applications, researchers most commonly measure three heads (lateral, long and medial) of the TB, (2) $\mathrm{Ag} / \mathrm{AgCl}$ electrodes are utilised more frequently than all other types of electrodes, such as gold, silver, nickel silver, and polycarbonate electrodes, for sEMG, and (3) a 10mm electrode size and a 20-mm interelectrode distance are commonly used to measure the sEMG activity of the TB with bipolar $\mathrm{Ag} / \mathrm{AgCl}$ electrodes. However, due to motion of the subject, it remains challenging to use the sEMG recording technique on the TB for the rehabilitation of patients and as the analysis of the sports performance of athletes. Although several protocols have been established for the use of the sEMG recording technique on TB during dynamic contraction, their use for real-time analysis may still be compromised by the factors highlighted in the present study.

\section{Acknowledgments}

The authors would like to thank to all of researchers of the AI-Rehab Research Group, UniMAP for their cooperation.

\section{References}

[1] C. Frigo and P. Crenna, Multichannel SEMG in clinical gait analysis: A review and state-of-the-art, Clinical biomechanics (Bristol, Avon) 24 (2009), 236-245.

[2] J.V. Basmajian and C.J. De Luca, Muscles alive: Their functions revealed by electromyography, Williams \& Wilkins, NY, USA, 1985.

[3] E. Swinnen, J.-P. Baeyens, R. Meeusen and E. Kerckhofs, Methodology of electromyographic analysis of the trunk muscles during walking in healthy subjects: A literature review, Journal of Electromyography and Kinesiology 22 (2012), $1-12$.

[4] M.A.M. Benhamou, M. Revel and C. Vallee, Surface electrodes are not appropriate to record selective myoelectric activity of splenius capitis muscle in humans, Experimental Brain Research 105 (1990), 432-438.

[5] R. Merletti and D. Farina, Analysis of intramuscular electromyogram signals, Philosophical Transactions of the Royal Society A: Mathematical, Physical and Engineering Sciences 367 (2009), 357-368.

[6] M. Ortiz-Catalan, R. Branemark, B. Hakansson and J. Delbeke, On the viability of implantable electrodes for the natural control of artificial limbs: Review and discussion, BioMedical Engineering OnLine 11 (2012), 33.

[7] D.P. Ferris, H.J. Huang and P.-C. Kao, Moving the arms to activate the legs, Exercise and Sport Sciences Reviews 34 (2006), 113-120

[8] M. Pijnappels, I. Kingma, D. Wezenberg, G. Reurink and J. Dieën, Armed against falls: The contribution of arm movements to balance recovery after tripping, Experimental Brain Research 201 (2010), 689-699.

[9] S. Salmons, Muscle In Gray's anatomy, P.L. B.L. Williams, M.M. Berry, P. Collins, M. Dyson, J.E. Dussek, M.W.J. Ferguson, ed. Churchii Livingstone, NY, USA, 1995, pp. 737-900.

[10] G.J. Tortora, Principles of human anatomy, Edited by Hoboken, NJ: Wiley and Sons, 2005.

[11] K.M.V.D. Graaff, Human anatomy, Edited by Dubuque IA McGraw-Hill, 2002.

[12] P. Williams, R. Warwick, M. Dyson and L. Bannister, Gray's anatomy 37th ed, Edinburgh, Scotland: Churchill Livingstone, 1989, 1356-1394.

[13] T. Pickering and R. Howden, Muscle, Grays' anatomy, 15th edition Facsimile;1901 (Reproduced by Barnes and Nobles), 1995.

[14] D. Landin and M. Thompson, The shoulder extension function of the triceps brachii, Journal of Electromyography and Kinesiology 21 (2011), 161-165.

[15] K.L. Moore and A.F. Dalley, Clinically oriented anatomy, Lippincott Williams \& Wilkins, NY, USA, 1992.

[16] T.S. Buchanan, D.P. Almdale, J.L. Lewis and W.Z. Rymer, Characteristics of synergic relations during isometric contractions of human elbow muscles, Journal of Neurophysiology 56 (1986), 1225-1241 
[17] M.W.R. Holmes and P.J. Keir, Posture and hand load alter muscular response to sudden elbow perturbations, Journal of Electromyography and Kinesiology 22 (2012), 191-198.

[18] W. Hollinshead, Anatomy for surgeons: The back and limbs, Harper \& Row, NY, USA, 1982

[19] Seniam, Surface ElectroMyoGraphy for Non-Invasive Assesment of Muscle (SENIAM), http://www.seniam.org (2010).

[20] K. Emery and J. Côté, Repetitive arm motion-induced fatigue affects shoulder but not endpoint position sense, Experimental Brain Research 216 (2012), 553-564.

[21] M. Serrao, A. Ranavolo, O.K. Andersen, R. Don, F. Draicchio, C. Conte, R. Di Fabio, A. Perrotta, M. Bartolo, L. Padua, V. Santilli, G. Sandrini and F. Pierelli, Reorganization of multimuscle and joint withdrawal reflex during arm movements in post-stroke hemiparetic patients, Clinical Neurophysiology 123 (2012), 527-540.

[22] Y. Fang, J.J. Daly, J. Sun, K. Hvorat, E. Fredrickson, S. Pundik, V. Sahgal and G.H. Yue, Functional corticomuscular connection during reaching is weakened following stroke, Clinical neurophysiology 120 (2009), 994-1002.

[23] R. Barker, S. Brauer and R. Carson, Training-induced changes in the pattern of triceps to biceps activation during reaching tasks after chronic and severe stroke, Experimental Brain Research 196 (2009), 483-496.

[24] L. Li, K.Y. Tong, X.L. Hu, L.K. Hung and T.K.K. Koo, Incorporating ultrasound-measured musculotendon parameters to subject-specific EMG-driven model to simulate voluntary elbow flexion for persons after stroke, Clinical Biomechanics 24 (2009), 101-109.

[25] Y.J.M. Janssen-Potten, H.A.M. Seelen, H.M.H. BongersJanssen and L.H.V. van der Woude, Assessment of upper extremity muscle function in persons with tetraplegia, Journal of Electromyography and Kinesiology 18 (2008), 516-526.

[26] J.W. Rankin, A.M. Kwarciak, W.M. Richter and R.R. Neptune, The influence of wheelchair propulsion technique on upper extremity muscle demand: A simulation study, Clinical Biomechanics 27 (2012), 879-886.

[27] H.J. Huang and D.P. Ferris, Upper limb effort does not increase maximal voluntary muscle activation in individuals with incomplete spinal cord injury, Clinical neurophysiology 120 (2009), 1741-1749.

[28] N. Louis and P. Gorce, Surface electromyography activity of upper limb muscle during wheelchair propulsion: Influence of wheelchair configuration, Clinical Biomechanics 25 (2010), 879-885.

[29] S.M. Brændvik and K. Roeleveld, The role of co-activation in strength and force modulation in the elbow of children with unilateral cerebral palsy, Journal of Electromyography and Kinesiology 22 (2012), 137-144.

[30] J.P. Kuhtz-Buschbeck and B. Jing, Activity of upper limb muscles during human walking, Journal of Electromyography and Kinesiology 22 (2012), 199-206.

[31] M. Yung, S. Mathiassen and R. Wells, Variation of force amplitude and its effects on local fatigue, European Journal of Applied Physiology 112 (2012), 3865-3879.

[32] I. Bazzucchi, M.E. Riccio and F. Felici, Tennis players show a lower coactivation of the elbow antagonist muscles during isokinetic exercises, Journal of Electromyography and Kinesiology 18 (2008), 752-759.

[33] S. Rota, C. Hautier, T. Creveaux, S. Champely, A. Guillot and I. Rogowski, Relationship between muscle coordination and forehand drive velocity in tennis, Journal of Electromyography and Kinesiology 22 (2012), 294-300.

[34] I. Rogowski, T. Creveaux, A. Faucon, S. Rota, S. Champely, A. Guillot and C. Hautier, Relationship between muscle coordination and racket mass during forehand drive in tennis, European Journal of Applied Physiology 107 (2009), 289-298.

[35] O.P. Neto and M. Magini, Electromiographic and kinematic characteristics of Kung Fu Yau-Man palm strike, Journal of Electromyography and Kinesiology 18 (2008), 1047 1052.

[36] A.M. VencesBrito, M.A. Rodrigues Ferreira, N. Cortes, O. Fernandes and P. Pezarat-Correia, Kinematic and electromyographic analyses of a karate punch, Journal of Electromyography and Kinesiology 21 (2011), 1023-1029.

[37] J. Frère, B. Göpfert, J. Slawinski and C. Tourny-chollet, Effect of the upper limbs muscles activity on the mechanical energy gain in pole vaulting, Journal of Electromyography and Kinesiology 22 (2012), 207-214.

[38] I. Stirn, T. Jarm, V. Kapus and V. Strojnik, Evaluation of muscle fatigue during 100-m front crawl, European Journal of Applied Physiology 111 (2011), 101-113.

[39] Y. Ikuta, Y. Matsuda, Y. Yamada, N. Kida, S. Oda and T. Moritani, Relationship between decreased swimming velocity and muscle activity during 200-m front crawl, European Journal of Applied Physiology 112 (2012), 3417-3429.

[40] K.R. Lohse, D.E. Sherwood and A.F. Healy, How changing the focus of attention affects performance, kinematics, and electromyography in dart throwing, Human Movement Science 29 (2010), 542-555.

[41] H.J. Hermens, B. Freriks, C. Disselhorst-Klug and G. Rau, Development of recommendations for SEMG sensors and sensor placement procedures, Journal of Electromyography and Kinesiology 10 (2000), 361-374.

[42] N.A. Dimitrova, G.V. Dimitrov and V.N. Chihman, Effect of electrode dimensions on motor unit potentials, Medical Engineering \& Physics 21 (1999), 479-485.

[43] C.M. Sommerich, S.M.B. Joines, V. Hermans and S.D. Moon, Use of surface electromyography to estimate neck muscle activity, Journal of Electromyography and Kinesiology $\mathbf{1 0}$ (2000), 377-398.

[44] A. Perotto ed. Anatomical guide for the electromyographer: The limbs and trunk, Charles $\mathrm{C}$ Thomas Publisher, Springfield, 1996.

[45] K.G. Cram JR, J. Holtz and M.D. Gaithersburg, ed. Introduction to surface electromyography, Aspen Publishers, Inc., 1998.

[46] L. Mesin, R. Merletti and A. Rainoldi, Surface EMG: The issue of electrode location, Journal of Electromyography and Kinesiology 19 (2009), 719-726.

[47] G. Kamen and D. Gabriel, Essentials of Electromyography. Human Kinetics, 2010.

[48] J.H. Blok and D.F. Stegeman, Simulated bipolar sEMG characteristics (Edited by Hermens HJ and Freriks B: Roessingh Research and Development, Enschede, The Netherlands SENIAM deliverable 5), (1997), 60-70.

[49] R. Merletti, A. Botter, A. Troiano, E. Merlo and M.A. Minetto, Technology and instrumentation for detection and conditioning of the surface electromyographic signal: State of the art, Clinical Biomechanics 24 (2009), 122-134.

[50] R. Merletti, Standards for reporting EMG data, Journal of Electromyography and Kinesiology 7 (1997), I-II. 
[51] D. Farina, Counterpoint: Spectral properties of the surface emg do not provide information about motor unit recruitment and muscle fiber type, Journal of Applied Physiology 105 (2008), 1673-1674

[52] V. von Tscharner and B.M. Nigg, Point: Counterpoint: Spectral properties of the surface EMG can characterize/do not provide information about motor unit recruitment strategies and muscle fiber type, Journal of Applied Physiology 105 (2008), 1671-1673.

[53] D. Farina, Interpretation of the Surface Electromyogram in Dynamic Contractions, Exercise and Sport Sciences Reviews 34 (2006), 121-127.

[54] D. Farina, R. Merletti and R.M. Enoka, The extraction of neural strategies from the surface EMG, Journal of Applied Physiology 96 (2004), 1486-1495.
[55] R. Merletti, A. Rainoldi and D. Farina, Surface electromyography for noninvasive characterization of muscle, Exercise and Sport Sciences Reviews 29 (2001), 20-25.

[56] H. Christensen, K. Søgaard, B.R. Jensen, L. Finsen and G. Sjøgaard, Intramuscular and surface EMG power spectrum from dynamic and static contractions, Journal of Electromyography and Kinesiology 5 (1995), 27-36.

[57] S. Shankar, R.E. Gander and B.R. Brandell, Changes in the myoelectric signal (MES) power spectra during dynamic contractions, Electroencephalography and Clinical Neuro physiology 73 (1989), 142-150.

[58] R. Merletti, A. Botter, C. Cescon, M.A. Minetto and T.M.M. Vieira, Advances in surface EMG: Recent progress in clinical research applications, Critical Reviews in Biomedical Engineering 38 (2010), 347-379. 

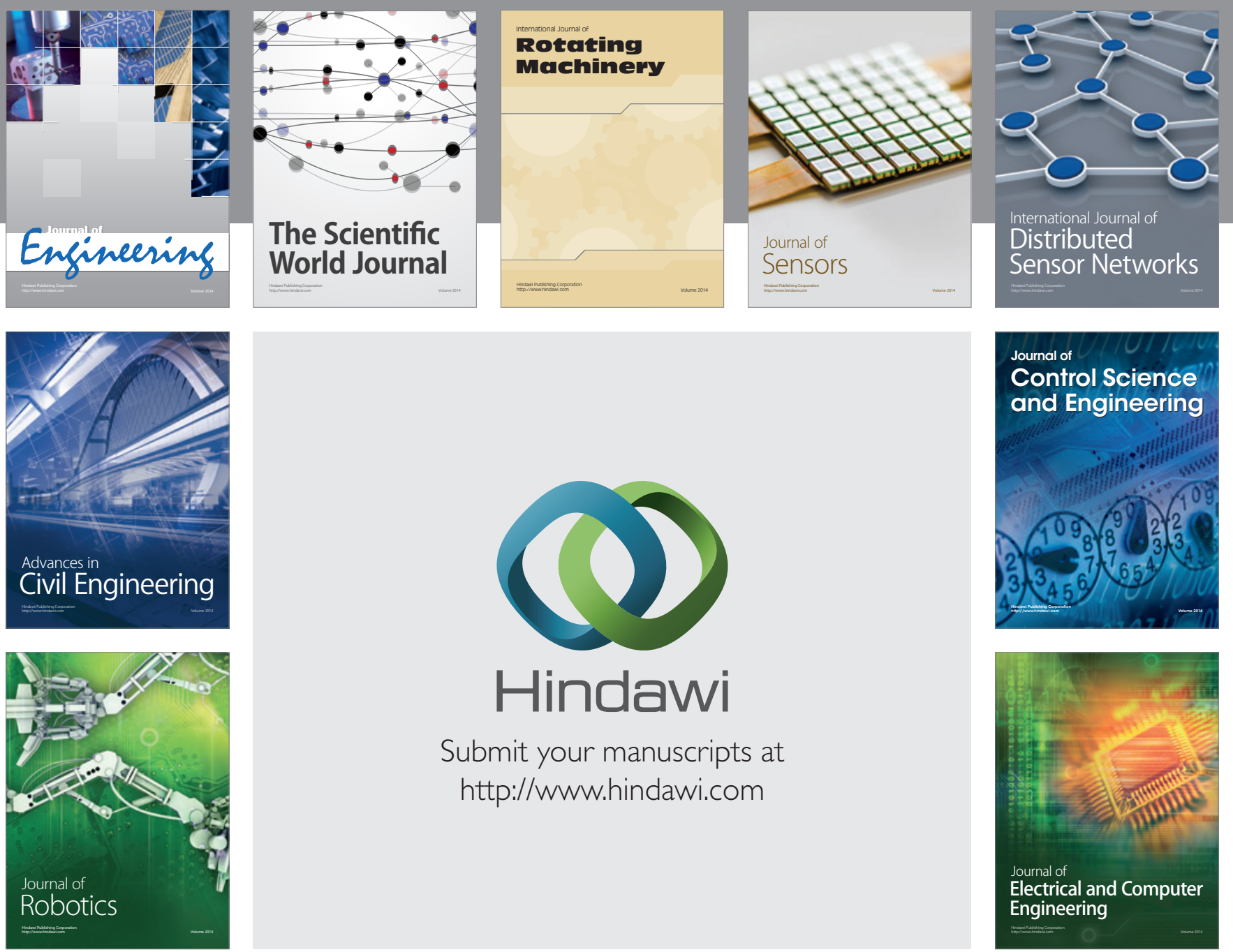

Submit your manuscripts at

http://www.hindawi.com
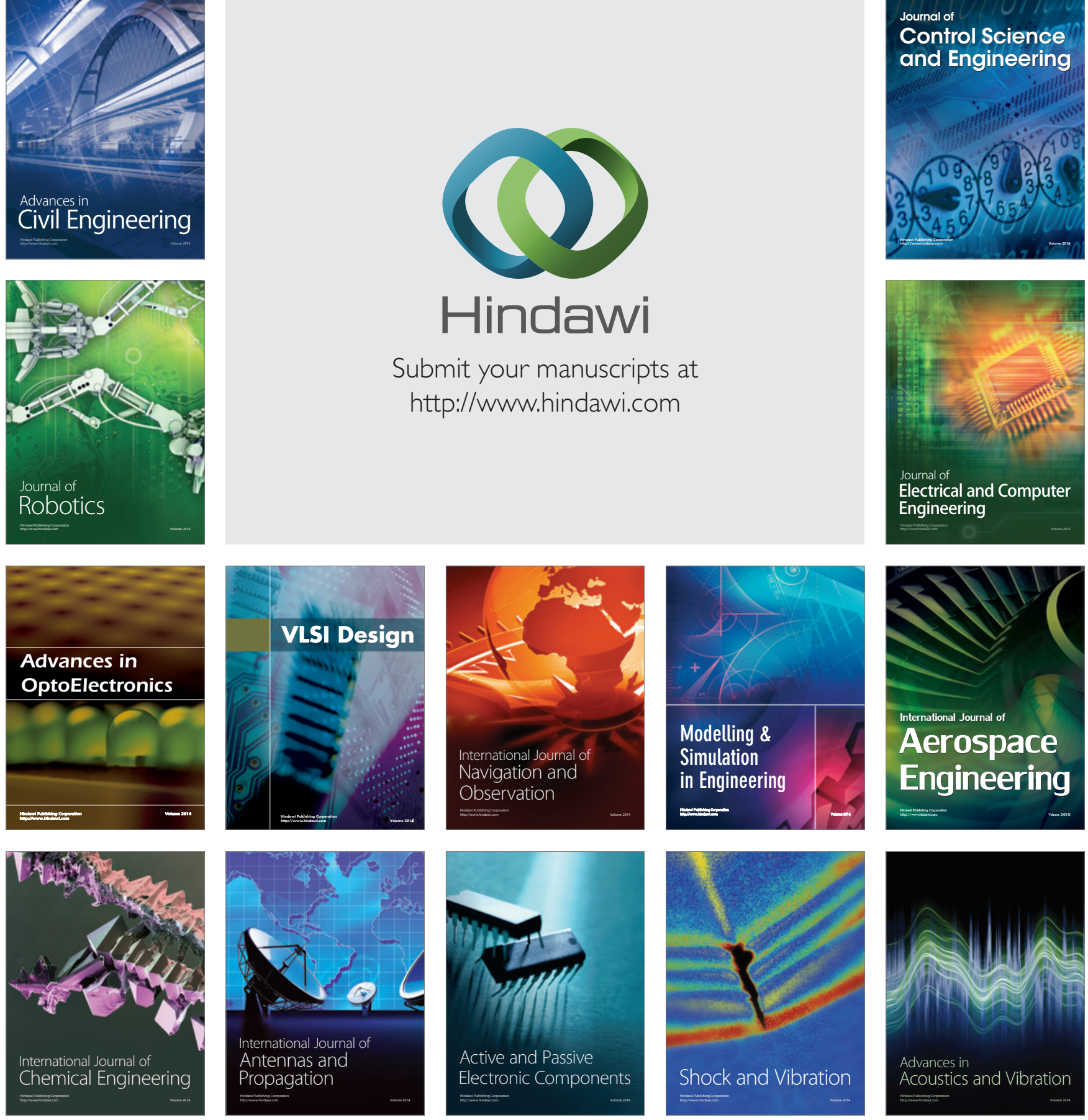